\title{
Correction to: A glassy carbon electrode modified with N-doped carbon dots for improved detection of hydrogen peroxide and paracetamol
}

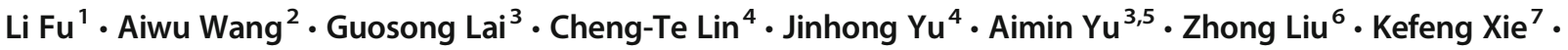 \\ Weitao $\mathrm{Su}^{1}$
}

Published online: 11 June 2019

(C) Springer-Verlag GmbH Austria, part of Springer Nature 2019

\section{Correction to: Microchimica Acta (2018) 185: 87}

https://doi.org/10.1007/s00604-017-2646-9

The authors of "A glassy carbon electrode modified with Ndoped carbon dots for improved detection of hydrogen peroxide and paracetamol (Microchimica Acta 185, no. 2 (2018): 87)" wish to replace the incorrect images of Fig. 1C, 1D shown below. Unfortunately in original work, the XPS elemental survey of the NCDs was wrongly given.

The correct images of XPS data for N doped carbon dots showed below.

The online version of the original article can be found at https://doi.org/ $10.1007 / \mathrm{s} 00604-017-2646-9$

$\mathrm{Li} \mathrm{Fu}$

fuli@hdu.edu.cn

Weitao Su

suweitao@hdu.edu.cn

1 College of Materials and Environmental Engineering, Hangzhou Dianzi University, Hangzhou 310018, People's Republic of China

2 Center of Super-Diamond and Advanced Films (COSDAF) and Department of Physics and Materials Science, City University of Hong Kong, Hong Kong, Hong Kong

3 Hubei Collaborative Innovation Center for Rare Metal Chemistry, Hubei Key Laboratory of Pollutant Analysis \& Reuse Technology, Department of Chemistry, Hubei Normal University, Huangshi 435002, People's Republic of China
4 Key Laboratory of Marine Materials and Related Technologies, Zhejiang Key Laboratory of Marine Materials and Protective Technologies, Ningbo Institute of Materials Technology and Engineering, Chinese Academy of Sciences, Ningbo 315201, China

5 Department of Chemistry and Biotechnology, Faculty of Science, Engineering and Technology, Swinburne University of Technology, Hawthorn, VIC 3122, Australia

6 Key Laboratory of Comprehensive and Highly Efficient Utilization of Salt Resources, Qinghai Institute of Salt Lakes, Chinese Academy of Sciences, Xining 810008, People's Republic of China

State Key Laboratory of Plateau Ecology and Agriculture, Qinghai University, Xining 810016, People's Republic of China 


\section{Correct Fig 1}
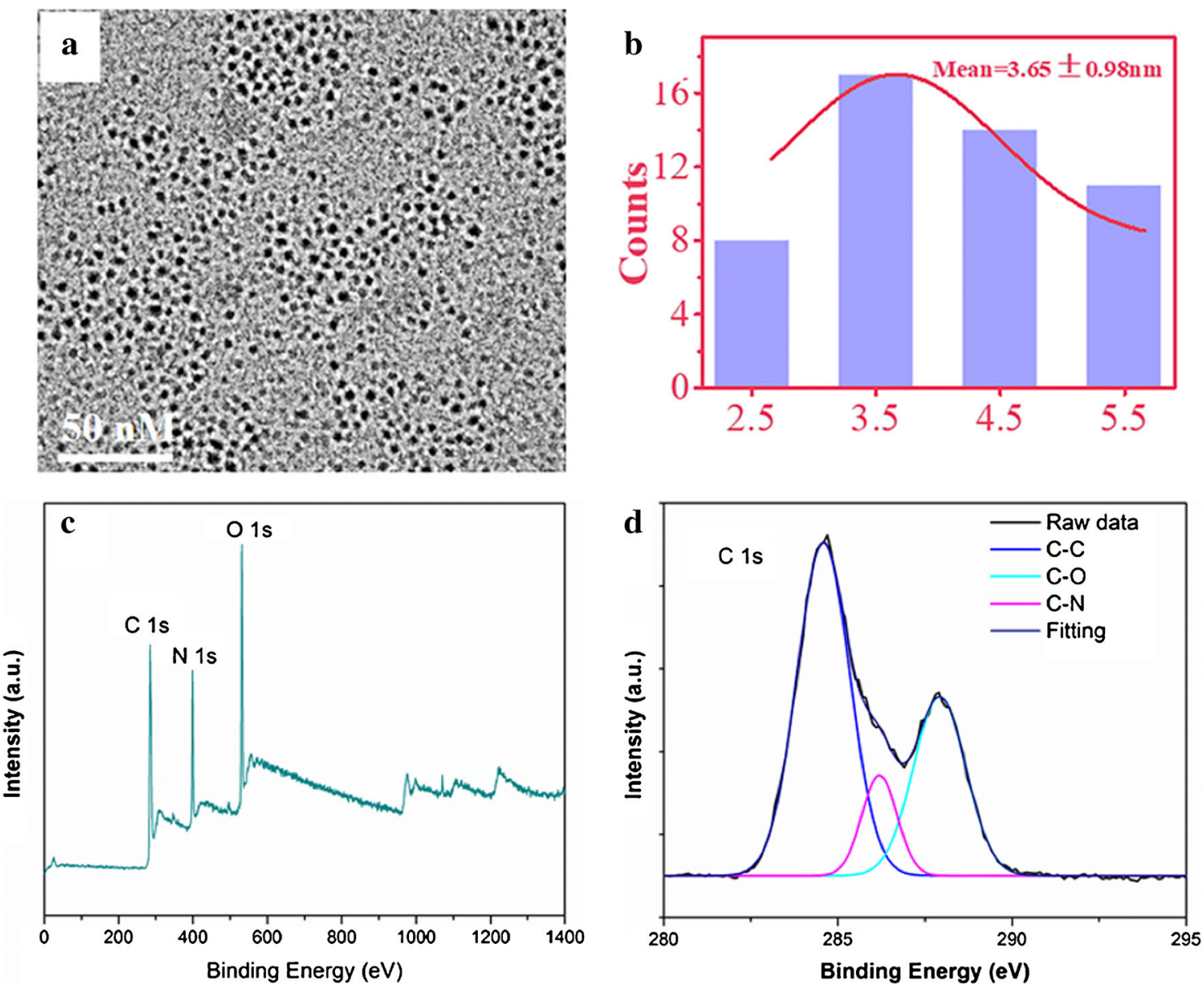

In the Results and discussion:

In the Conclusion:

"The percentages of C, N, O are 59.1\%, $19.5 \%$ and $20.71 \%$ " should be revised to "The percentages of $\mathrm{C}, \mathrm{N}, \mathrm{O}$ are $33.2 \%$, $28.5 \%$ and $38.3 \% "$.

"The peaks located at $285.2 \mathrm{eV}$ and $287.2 \mathrm{eV}$ are assigned to $\mathrm{N}-\mathrm{sp}^{2}$ carbon and $\mathrm{N}-\mathrm{sp}^{3}$ carbon" should be revised to "The peaks located at $286.2 \mathrm{eV}$ and 287.9 is assigned to $\mathrm{C}-\mathrm{N}$ and CO bonding".

"A room-temperature" should be revised to "A lowtemperature".

Publisher's note Springer Nature remains neutral with regard to jurisdictional claims in published maps and institutional affiliations. 